\title{
An Improved Optimization Approach Based on Competitive Algorithm Qi-Mei WANG ${ }^{1, a}$, Shu-Hui XU², Zhi-Hong YANG ${ }^{3, b, *}$ \\ ${ }^{1}$ School of Business,Northeast Normal University, Changchun, China \\ ${ }^{2,3}$ Institute of Industrial Engineering, School of Mechanical Engineering, Shandong University, Jinan, China
}

ayecong124@163.com, byzh70@sdu.edu.cn

${ }^{*}$ Corresponding author

Keywords: Competitive algorithm, Optimization, Nelder-mead simplex method.

\begin{abstract}
In the paper, we developed an improved approach for the imperialist competitive algorithm to help the original algorithm escape from local optimal area and obtain better performance. The Nelder-Meand simplex method is also introduced to execute alternately with the original procedures of the algorithm. The proposed approach is tested on twelve widely-used benchmark functions to check its validity. Compared with other relative studies, the proposed approach shows faster convergence rate, better search ability, and better stability than the original algorithm and other relative methods.
\end{abstract}

\section{Introduction}

Optimization aims to obtain the best result under given circumstances, which is employed in almost every branch of science and technology. Usually, optimization tasks can be reduced to the problems of finding the minimum value or the maximum value for a function of one or more variable parameters [1]. Actually, finding the maximum value or the minimum value is entirely equivalent since one can be converted to the other by changing the sign of the function. In recent decades, using heuristic algorithms to deal with such problems has attracted more and more attention [2]. Imperialist competitive algorithm (ICA) is a new population-based heuristic algorithm proposed by Atashpaz-Gargari and Lucas in 2007, inspired by the colonial phenomenon in human society and history [3]. Unfortunately, similar to other population-based heuristic algorithms, it also often suffers premature convergence and falls into local optimal area, especially when the problem is complicated, high-dimensional or multi-peak [4].

Several improvement approaches have been proposed to enhance the algorithm's performance. All the improvement approaches mentioned above are focused on how to modify the detail rules of the basic ICA. They make the algorithm becomes very complicated while geting some improvements. Meanwhile, experimental results show that these approaches are efficient, but not good enough. Under such a background, seeking a simpler and more efficient improvement strategy becomes the aim of our research.

The Nelder-Mead simplex method (NM), firstly proposed by Nelder and Mead [5], is particularly designed for traditional unconstrained minimization scenarios. This method is powerful. It does not need the function's derivatives which cannot be always precisely obtained in many problems. This feature makes it so popular and has been widely used in many problems. However, this method is highly sensitive to the choice of initial points. If the initial points are inappropriate, it is easy to fail to obtain the global optimum or cost too much time.

The ICA converges fast, but its local search ability is relatively poor. The NM is sensitive to the initial points, and as a local search method, it has good exploitation ability. Meanwhile, the NM has been introduced into many different heuristic algorithms to enhance their ability. In this paper, a new hybrid algorithm is proposed by introducing the NM into the ICA to have their different characteristics. 


\section{Imperialist Competitive Algorithm}

Similar with other heuristic algorithms, ICA also starts with a randomly generated population which is formed by $\mathrm{N}$ individuals. Here an individual is represented using a concept of 'country'. For a $N_{v a r}$ dimensional optimization problem, a country is a one dimensional array which includes $N_{\text {var }}$ elements. Each element corresponds to one parameter and is randomly generated in the corresponding range, as: country $=\left[p_{1}, p_{2}, p_{3}, \ldots, p_{N v a r}\right]$. After, the cost of each country is calculated on the basis of the cost function, cost $=f($ country $)=f\left(p_{1}, p_{2}, p_{3}, \ldots, p_{N v a r}\right)$.

Then, some best countries are selected from the population and to be regarded as imperialists. Meanwhile, the rest countries are regarded as colonies. After that, the cost of each imperialist is normalized by Eq.(1), where $c_{n}$ and $C_{n}$ represent the cost and the normalized cost of the $n$th imperialist, respectively. Then, the normalized power of every imperialist is calculated based on its normalized cost, by Eq.(2), where $N_{i m p}$ represents the total number of the imperialists and $p_{n}$ represents the normalized power of the $n$th imperialist.

$$
\begin{gathered}
C_{n}=c_{n}-\max _{i}\left\{c_{i}\right\} \\
p_{n}=\left|\frac{C_{n}}{\sum_{i=1}^{N_{\text {inp }}} C_{i}}\right|
\end{gathered}
$$

The colonies are randomly allocated to the imperialists, to form empires. The imperialist with larger power value can get more colonies and every colony should be allocated to one and only one imperialist. Eq.(3) gives the calculation method, where $N_{.} C_{\cdot n}$ and $N_{c o l}$ are the number of the colonies allocated to the $n$th imperialist and the total number of the colonies, respectively. One imperialist country and the colonies allocated to it constitute one empire.

$$
N \cdot C_{\cdot_{n}}=\operatorname{round}\left\{p_{n} \square N_{c o l}\right\}
$$

Then, within every empire group, colonies are moved towards the imperialist. A random amount of deviation is added to the movement direction, to provide better exploration ability for the algorithm. This process is called 'assimilation', illustrated by Fig. 1, where $x$ represents the final displacement; $d$ represents the distance between the colony and the relevant imperialist; $\beta$ is a number which is greater than 1 , used to guarantee the colony can get closer to the imperialist from both sides; $\gamma$ is a parameter whose value determines the range of the deviation; $\theta$ represents the additional deviation angle. Usually, when $\beta$ is set to a value of about 2 and $\gamma$ is set to a value of about $\pi / 4$ (rad), the ICA can obtain a relatively good performance.

Meanwhile, a part of colonies are randomly selected and then replaced with an equal number of new randomly generated countries. This process is called 'revolution', similar to the mutation in the genetic algorithm. The rate of this part colonies occupied in all the colonies is called 'revolution rate'. Revolution operation enhances the ability to escape from local optima and then avoid premature convergence.

In assimilation and revolution process, if a colony becomes better than the imperialist it belongs to, then their roles will be exchanged; if two imperialists become very close to each other, the two corresponding empires will be united to one empire, in which the better one of the two old imperialists will be the new imperialist and take over all the colonies of the two old empires.

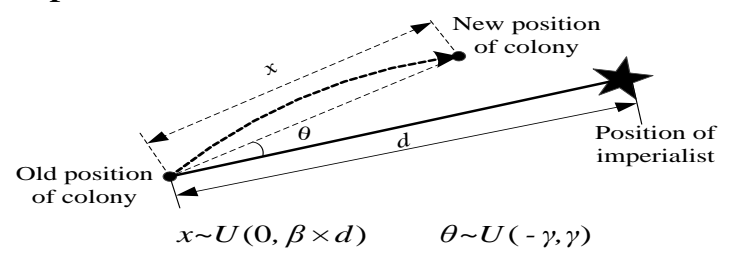

Figure 1 The assimilation process 


\section{Nelder-mead Simplex Method}

The NM goes as follows: for an $N_{v a r}$ dimensional minimization function, firstly, $N_{v a r}+1$ initial vertex points are randomly generated in the search space. In every iteration, the point with highest cost value (the worst point) will be replaced by a new better point. The new point is generated by one of four different operators: reflection, expansion, contraction and shrinkage. Which operator will be used depends on the specific situation, outlined below in detail. Fig. 2 shows the four operators using a two-dimensional case.

In each iteration, the cost of every vertex point is evaluated. Suppose that the point with the highest cost is marked as $\mathrm{P}_{\text {high }}$, the point with the lowest cost is marked as $\mathrm{P}_{\text {low }}$, the point with the second highest cost is marked as $\mathrm{P}_{\text {sec }}$. And their cost values are marked as $f_{\text {high }}, f_{\text {low }}, f_{\text {sec }}$, respectively.

\section{The Approach of Introducing the NM into the ICA}

Though the implementation processes of both the two algorithms are clear, in order to get satisfactory performance, how to combine them together is the key and difficult point. The approach we proposed goes as follows: For an $N_{v a r}$ dimensional minimization function, the size of the initial population of the ICA is set more than the $N_{v a r}$ (about 3 4 times as big as $N_{v a r}$ ). In each iteration of the ICA, after finished the assimilation process, the revolution process and the competition process, the number of colonies in every empire is examined. The Nelder-Mead simplex method (NM) will be used on those empires which control more than $N_{v a r}$ colonies. Firstly, the costs of all the colonies in the empire are evaluated and sorted. $N_{v a r}$ best colonies among them are selected out, added with the imperialist of the empire, consist of an initial simplex. Then, an iteration of the Nelder-Mead simplex method (NM) is executed. One (in the case of reflection, expansion or contraction) or more (in the case of shrink) points would be improved by the NM method. After then, the cost of every point of the simplex is evaluated and sorted again. The best one will be used as the imperialist of the empire. The others are used to replace the $N_{v a r}$ worst colonies, illustrated as Fig. 3. at last, the ICA continues with a new iteration.

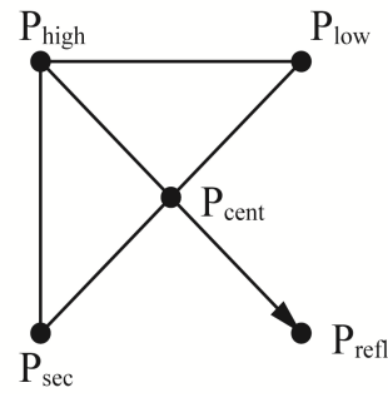

(a) reflection

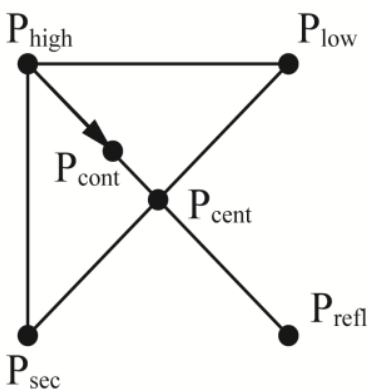

(d) contraction ( $\mathrm{P}_{\text {high }}$ is better than $\left.\mathrm{P}_{\text {refl }}\right)$

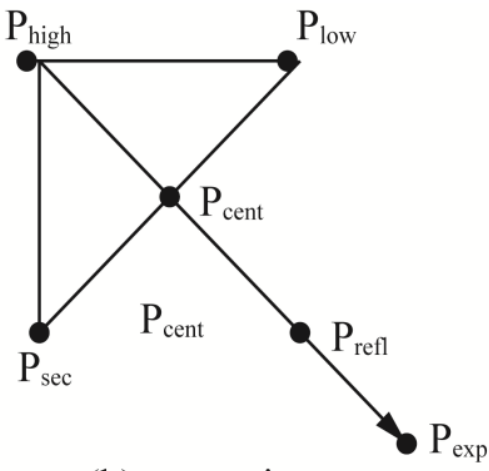

(b) expansion

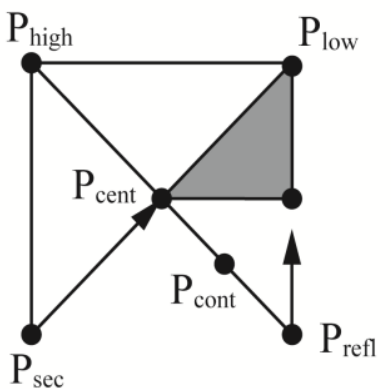

(e) shrink (contraction failed, and $\mathrm{P}_{\text {refl }}$ is better than $\mathrm{P}_{\text {high }}$ )

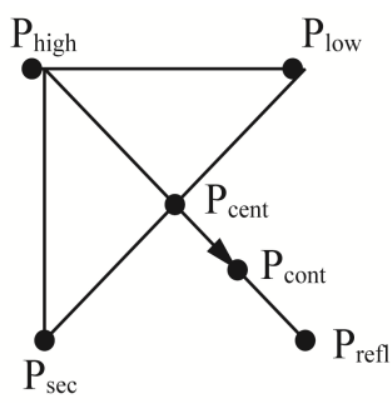

(c) contraction $\left(\mathrm{P}_{\text {refl }}\right.$ is better than $\mathrm{P}_{\text {high }}$ )

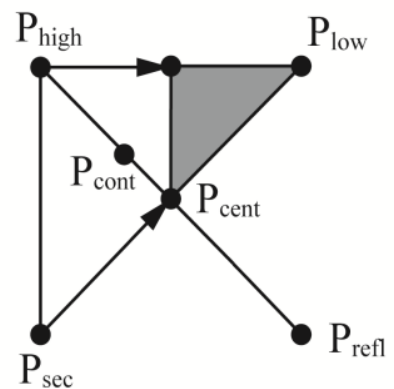

(f) shrink (contraction failed, and $\mathrm{P}_{\text {high }}$ is better than $\mathrm{P}_{\text {refl }}$ )

Figure 2 The four basic operators in the NM 


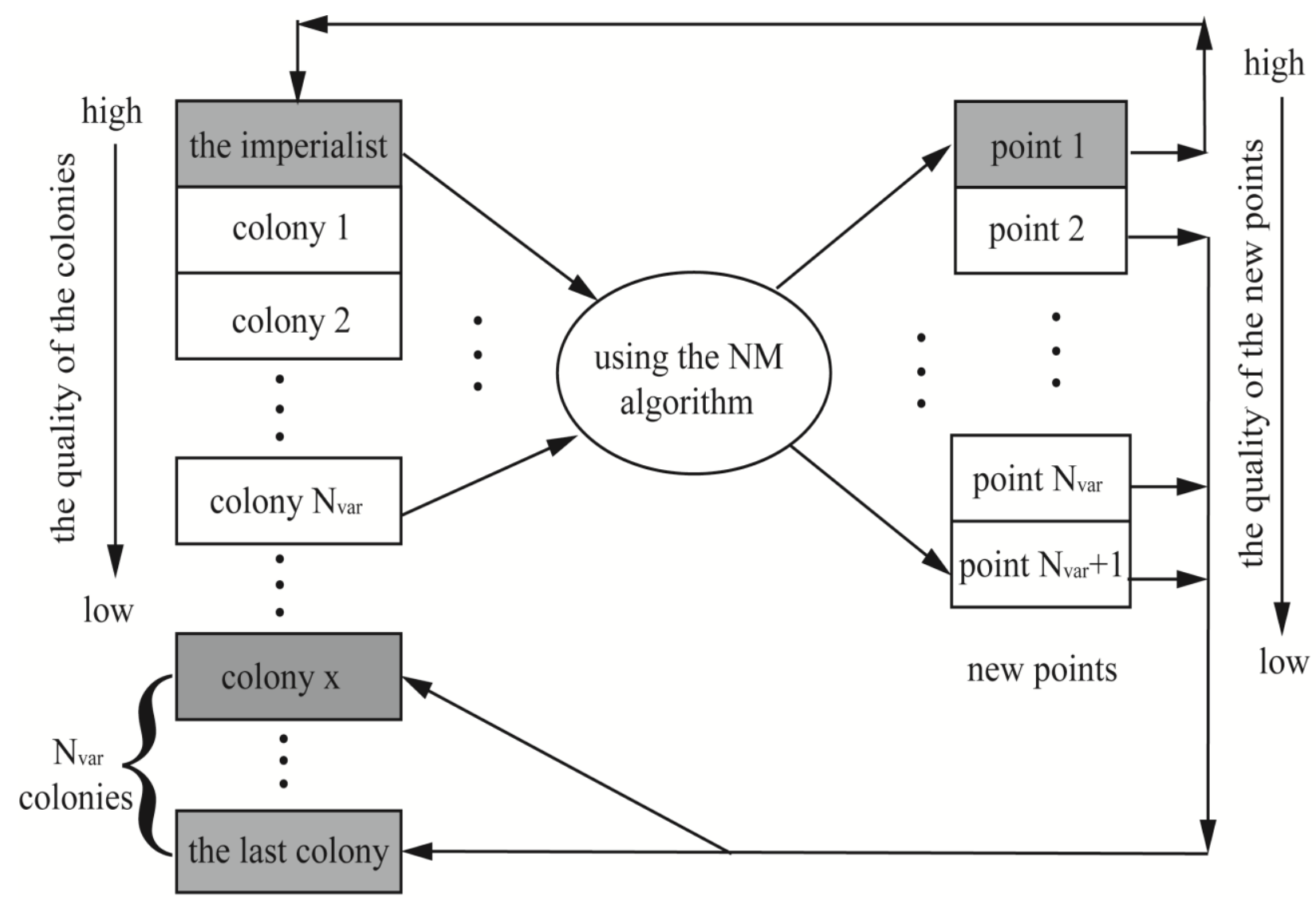

an empire,the colonies are sorted according to their qualities.

Figure 3 The approach to introduce the NM into the basic ICA

\section{Results and Discussions}

In order to study the behaviors of the two algorithms more clearly, on every function, both the two algorithms were run once on every function using same initial empires (same initial imperialists, same initial colonies and same subordinate relations between the imperialists and the colonies, used to eliminate the effects may be caused by the difference of initial empire).

The convergence curves of the two algorithms started with same initial empires on every function is shown in Fig.4. It is observed that, the convergence curves of the improved ICA we proposed are steeper, means that the improved ICA has faster convergence speed. From the convergence curves of $f_{4}, f_{5}, f_{6}, f_{7}, f_{8}, f_{9}, f_{10}$, and $f_{12}$, it can be observed that, falling into local optimum is a very serious problem for the basic ICA while this problem is milder for the improved ICA. On these functions, the basic ICA falls into local optimum area in the early or middle stage of the iteration process, the convergence curves began to close to the horizontal, the quality of the best imperialist cannot be improved further, while in the improved ICA, the imperialist is improved continuously and greatly, reflected as the continuous decline of the convergence curves. The cause of the difference is that in the basic ICA, after several iterations, the colonies would gather around to the imperialist they belong to and almostdon't move at all. So finding a better country becomes very hard. While in the improved ICA, the NM works constantly at this stage using a mechanism different with the assimilation, thanks to its powerful local search ability, the countries can still be improved greatly. 


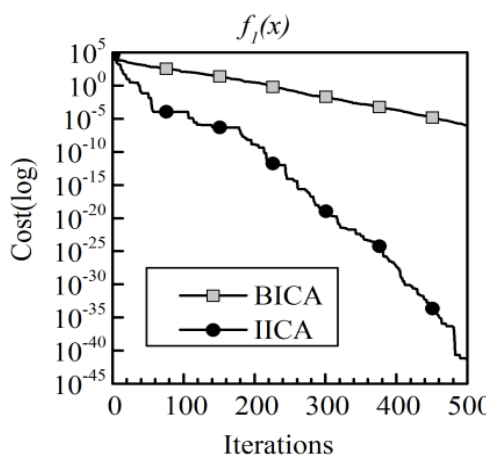

(a)

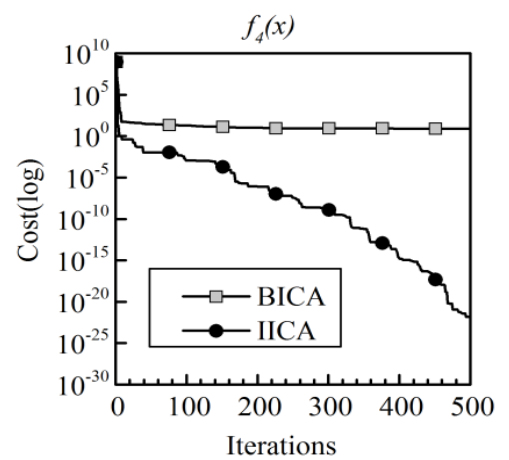

(d)

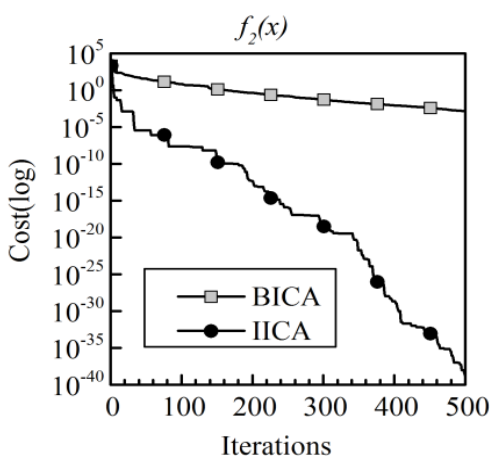

(b)

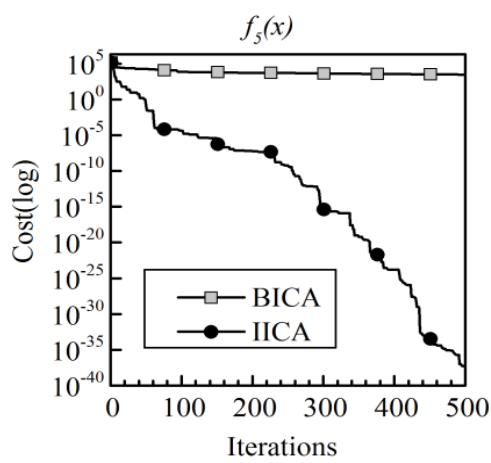

(e)

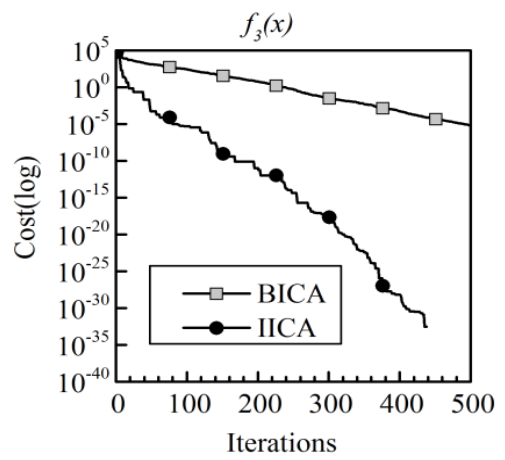

(c)

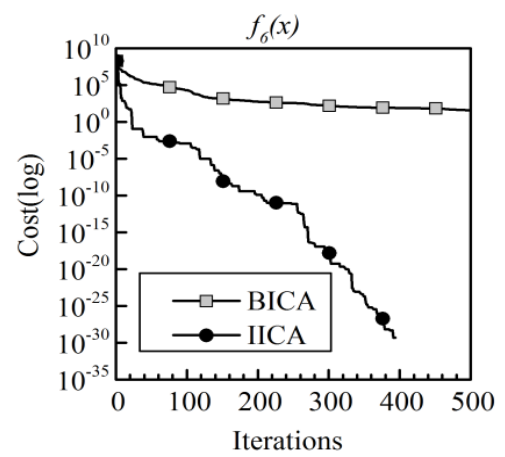

(f)

Figure 4 The convergence curves of the two algorithms on every function.

As mentioned before, various improved approaches for the basic ICA have been proposed. Among them, the most outstanding one is the improved approach proposed in [6] (abbreviated as CICA below). The CICA is tested on four 10-D benchmark functions. To compare its performance with the CICA, the improved ICA we proposed is also tested on these functions. For fair, the iterations number and the search ranges for every function in the improved ICA are set as same as those given. The experiment on every function is repeated 100 times.

The improved ICA we proposed shows better performance than the CICA on these four functions. On the former three functions, the improved ICA obtained the optimum with a success rate of one hundred percent. On Ackley function, though the improved ICA failed to find the optimum, it found a same result, which is better than the results obtained by the CICA, shows good stability.

\section{Summary}

By introducing the Nelder-Mead simplex method into the basic ICA, an improved ICA with simple structure and excellent performance is proposed in this paper. In the improved ICA, the Nelder-Mead simplex method is executed alternately with the original procedures of the algorithm and used to update the worst colonies. To check its performance, the improved ICA is tested on twelve widely-used 30-D benchmark functions. On every function, it obtains good results which are obviously better than that obtained by the basic ICA. Meanwhile, compared with the basic ICA, the improved ICA shows faster convergence rate, better global search ability and better stability. Furthermore, in order to compare it with another outstanding improved approach, the improved ICA is tested on four 10-D benchmark functions, and obtained better results. The applying of the improved ICA in diverse real world problems merits further research in our future work.

\section{References}

[1] A. Antoniou, W.-S. Lu, Practical optimization: algorithms and engineering applications, 2007 ed., Springer-Verlag, New York, 2007. 
[2] X. Yu, M. Gen, Introduction to evolutionary algorithms, Springer, London, 2010.

[3] E. Atashpaz-Gargari, C. Lucas, Imperialist competitive algorithm: An algorithm for optimization inspired by imperialistic competition, in: 2007 IEEE Congress on Evolutionary Computation, CEC 2007, IEEE Computer Society, Singapore 2007, pp. 4661-4667.

[4] M. Abdechiri, K. Faez, H. Bahrami, Adaptive imperialist competitive algorithm (AICA), in: Cognitive Informatics (ICCI), 2010 9th IEEE International Conference on, IEEE, 2010, pp. 940-945.

[5] D.M. Olsson, L.S. Nelson, The Nelder-Mead simplex procedure for function minimization, Technometrics, 17 (1975) 45-51.

[6] S. Talatahari, B. Farahmand Azar, R. Sheikholeslami, A. Gandomi, Imperialist competitive algorithm combined with chaos for global optimization, Communications in Nonlinear Science and Numerical Simulation, 17 (2012) 1312-1319. 